\title{
Zeno Effect in Parametric Down-Conversion
}

\author{
A. Luis* and J. Peřina ${ }^{\dagger}$ \\ Laboratory of Quantum Optics, Palacký University, 17. listopadu 50, 77207 Olomouc, Czech Republic
}

(Received 17 January 1996)

\begin{abstract}
Two examples are presented where the photon emission on spontaneous parametric down-conversion is prevented when attempts are made to infer the moment of emission. This inhibition is analyzed in terms of the disturbance caused on the system by the modifications that must be introduced in order to make possible such inference. [S0031-9007(96)00372-9]
\end{abstract}

PACS numbers: 42.50.Dv, 03.65.Bz

The Zeno effect refers to the inhibition of the isolated temporal evolution of a dynamical system when the observation of such evolution is attempted [1]. This observation is usually described by frequent measurements on the system performed in order to discover whether the initial state has changed or not. In the limit of very frequent measurements, continuous observation, or arbitrary high resolution, it may happen that the system is locked on its initial state and the evolution, which was the aim of the observation, is in fact inhibited and does not occur. It has been studied in atomic transitions [2], double-well potentials [3], and neutron spin dynamics [4], for example. Parallels can also be drawn with the interaction-free measurements [5].

In the first derivation the state reduction postulate of quantum mechanics was used [1]. According to this axiom, any measurement will abruptly change the state of the system under consideration so that it will be left in an eigenstate of the measured observable. This would link the Zeno effect directly with the quantum measurement theory. Since then, other approaches have been presented by means of purely dynamical terms without having to appeal to the reduction postulate $[3,4,6]$.

In most cases, in order to observe the intermediate stages of the evolution, it is necessary to modify the observed system in some way. The effect of these changes can be determined and understood only by a full quantum mechanical treatment of the whole process, which shows that even the most careful of all these observations inevitably leaves a trace on the observed system. In other words, the appearance of this effect can be attributed to this modification, or disturbance, which makes the observation possible, irrespective of whether the planned measurement is actually carried out or not.

Here we present two examples of Zeno effect that suit this framework. In both examples the process under observation is the simultaneous emission of a pair of photons by spontaneous parametric down-conversion in a nonlinear crystal. The entangled nature of this photon pair has been utilized hitherto in a number of fundamental experiments in quantum optics [7]. In our context, one of the emitted photons is evidence of the emission of the other. We will consider two different schemes using this fact in order to infer when the emission of the other photon takes place. In both cases these attempts lead to the inhibition of the emission.

Firstly, we briefly recall the isolated (or unobserved) dynamics of the system. A nonlinear crystal of length $L$ in Fig. 1(a) is pumped by a strong, classical, and coherent field (not shown in the figure) to produce pairs of signal and idler photons via spontaneous parametric down-conversion. Using the interaction representation with respect to the Hamiltonian of the free fields, this parametric process is described by the effective interaction Hamiltonian [8]

$$
H=\hbar g\left(a_{s}^{\dagger} a_{i}^{\dagger}+a_{s} a_{i}\right),
$$

where $a_{s}, a_{i}$ are the slowly varying annihilation operators for the signal and idler beams, and $g$, assumed to be real, is a coupling parameter depending on the pump field and the nonlinear characteristics of the medium. We have also assumed the frequency resonance condition $\omega_{p}=$ $\omega_{s}+\omega_{i}$, where $\omega_{p}, \omega_{s}$, and $\omega_{i}$ are the frequencies of the pump, signal, and idler beams, respectively. We will denote by $\tau$ the interaction time associated with the length
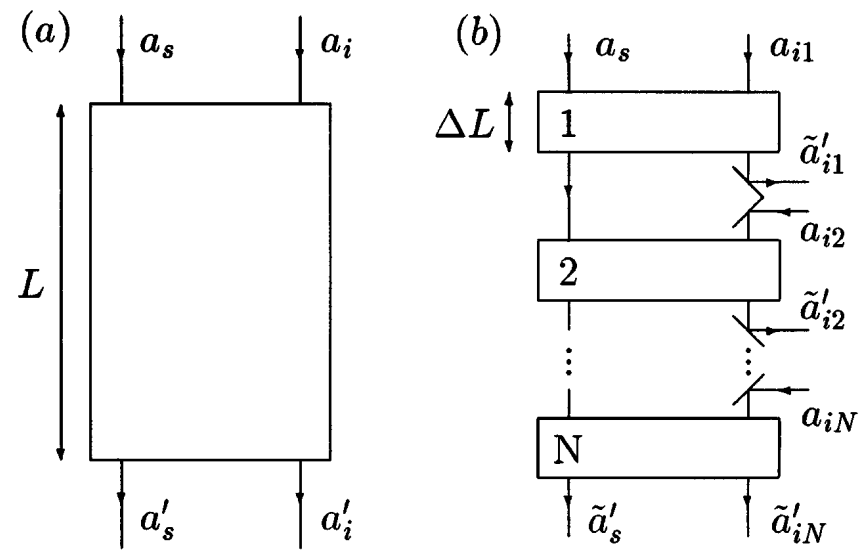

FIG. 1. (a) Outline of a parametric down-conversion scheme with a crystal of length $L$ showing the input $a_{s}, a_{i}$ and output $a_{s}^{\prime}, a_{i}^{\prime}$ complex amplitude operators for the signal and idler fields. (b) Modified scheme in order to infer the moment of emission and made of $N$ crystals of length $\Delta L=L / N$. After each piece, the output idler beams $\tilde{a}_{i k}^{\prime}$ are removed by mirrors inserted in the idler path and replaced by different input idler fields $a_{i k}$ in vacuum. In both cases, the beams have been represented parallel for simplicity. 
$L$ of the crystal. We focus on the generation of the signal beam from the vacuum. The interaction Hamiltonian (1) produces, after the interaction time $\tau$, the following general relation between the output complex amplitude $a_{s}^{\prime}$ for the signal field and the input signal and idler ones $a_{s}, a_{i}$ :

$$
a_{s}^{\prime}=\mu a_{s}+\nu a_{i}^{\dagger},
$$

where $\mu=\cosh g \tau$ and $\nu=-i \sinh g \tau$. Taking into account that before entering the crystal the signal and idler fields are in vacuum, we have that the probability of having $n$ output signal photons $P_{s}(n)$ is given by the Bose-Einstein distribution [8]

$$
P_{s}(n)=\frac{1}{\mu^{2}}\left(\frac{\mu^{2}-1}{\mu^{2}}\right)^{n},
$$

and the mean value of the number of signal photons is $\left\langle a_{s}^{\prime \dagger} a_{s}^{\prime}\right\rangle=\mu^{2}-1$. The following calculations can be carried out for an arbitrary value of $g \tau$, but the analysis becomes simpler if we consider the usual situation when $g \tau \ll 1$. In this short time approximation the probability of emission of more than one signal photon is negligible compared with that for one-photon emission, and we can simplify the final expressions retaining just the first power on $g \tau$. In this approximation the probability of having one signal photon, which coincides in this limit with the mean value of the number of photons, is

$$
P_{s}(1) \simeq\left\langle a_{s}^{\prime \dagger} a_{s}^{\prime}\right\rangle \simeq(g \tau)^{2} .
$$

The emission of the signal photon is always accompanied by the emission of the idler one. In principle, we can use this idler photon to detect the emission of the signal photon without disturbing or interrupting the signal path along the crystal.

The first modification of the previous scheme in order to infer when the emission of the signal photon occurs is shown in Fig. 1(b). The crystal is divided into $N$ equal parts of length $\Delta L=L / N$, being the associated interaction time $\Delta \tau=\tau / N$ within each part. We can assume that the signal beams of each part are perfectly superimposed and aligned, and that reflection at each piece can be avoided or made negligible, for instance, embedding the $N$ pieces in a linear medium with exactly the same refractive index. For simplicity we do not take into account some possible small imperfections such as partial misalignments and reflection losses which do not alter the substantial features of the process. On the other hand, the idler path is interrupted after each piece by means of mirrors, for instance. The output idler beams after each piece are completely removed from the idler path being replaced by new input idler beams which are in vacuum. This modification makes it possible to observe the $N$ output idler beams to detect the emission when it occurs, for instance, by means of $N$ photodetectors. Then, the moment of emission can be inferred with accuracy $\Delta \tau$, and the relative resolution is given by the number of pieces $\Delta \tau / \tau=1 / N$.

Next, we examine whether this arrangement has modified or disturbed the emission of the signal photon. As a consequence of the interruption of the idler path, a different vacuum mode $a_{i k}, k=1, \ldots, N$, is at the idler input of each of the $N$ crystals, and the signal output complex amplitude $\tilde{a}_{s}^{\prime}$ is given by

$$
\tilde{a}_{s}^{\prime}=\tilde{\mu}^{N} a_{s}+\tilde{\nu} \sum_{k=1}^{N} \tilde{\mu}^{N-k} a_{i k}^{\dagger},
$$

where $\tilde{\mu}=\cosh g \Delta \tau, \tilde{\nu}=-i \sinh g \Delta \tau$, and the validity of the boson commutation relations can be verified. We have that the probability of having $n$ signal photons at the output when all the incident fields are in vacuum is

$$
\tilde{P}_{s}(n)=\frac{1}{\tilde{\mu}^{2 N}}\left(\frac{\tilde{\mu}^{2 N}-1}{\tilde{\mu}^{2 N}}\right)^{n},
$$

and the mean value of the number of signal photons is given by $\left\langle\tilde{a}_{s}^{\prime \dagger} \tilde{a}_{s}^{\prime}\right\rangle=\tilde{\mu}^{2 N}-1$. In the short time approximation $g \tau \ll 1$, the probability of having one signal photon is given by

$$
\tilde{P}_{s}(1) \simeq\left\langle\tilde{a}_{s}^{\prime^{\dagger}} \tilde{a}_{s}^{\prime}\right\rangle \simeq N(g \Delta \tau)^{2}=(g \tau)^{2} / N,
$$

and the probability of having no signal photons can also be approximated by $\tilde{P}_{s}(0) \simeq 1-(g \tau)^{2} / N$. These expressions reflect the effect of the changes introduced in order to detect the emission of the photon. In comparison with the isolated evolution (4) we have that the probability of emission decreases when $N$ increases, being that this is the signature of the Zeno effect. Then, if the accuracy of the observation is increased by increasing $N$, the probability of the emission decreases at the same rate. In the limit $N \rightarrow \infty$ we would have a very frequent or continuous observation, which would allow an exact knowledge of the moment of emission. However, in this limit $N \rightarrow \infty$ we have $\tilde{P}_{s}(1) \rightarrow 0$ and $\tilde{P}_{s}(0) \rightarrow 1$, and there is no emission at all. We can note that whether the attempted measurement on the idler modes is actually made or not appears to make no difference. It is sufficient that it could be made.

This fact also indicates that this behavior can be traced back to the disturbance introduced in order to make possible the observation, and the effect must be caused by the removal of the output idler beams and their replacement by vacuum states. However, we might regard this inhibition as still paradoxical since the emission of the signal photon occurs spontaneously and at random, and the length covered by the signal beam within the nonlinear crystal is always the same $L$ and does not depend on $N$. Its path is not disturbed, and also the pumping is not affected. The parametric down-conversion is a completely quantum process having no classical analog, as it is reflected by the nonexistence of the GlauberSudarshan representation of the density matrix for all times. Therefore, the spontaneous process considered here is also fully quantum. Classically, there is no field generated in any of its realizations studied here, and then the classical theory cannot explain the different behavior of the emission. In order to understand their differences 
a quantum description of the field is needed. To this end, let us express the probability (4) of the unobserved system in the form

$$
P_{s}(1) \simeq\left\langle a_{s}^{\prime \dagger} a_{s}^{\prime}\right\rangle \simeq N^{2}(g \Delta \tau)^{2},
$$

which corresponds to divide the crystal of length $L$ into $N$ virtual parts of length $\Delta L=L / N$. These virtual parts could be made real if, in the scheme in Fig. 1(b), the idler beams were also perfectly aligned and superimposed instead of being interrupted, removing in this way the possibility of observing the emission. The dependence of (8) on $N$ in comparison with that in (7) recalls the superradiance effect [8]. We may say that for the unobserved system the emission is a cooperative effect of the $N$ parts, being proportional to $N^{2}$, whereas for the observed system we have an ordinary spontaneous emission from the $N$ pieces being then proportional to $N$. This indicates that in the first case the $N$ emissions are mutually coherent and the final result arises from the superposition of probability amplitudes, while in the second case the emissions are mutually incoherent and we have just the addition of probabilities.

A heuristic argument can be given to provide a simple insight on this different behavior if we consider these spontaneous emissions as stimulated by the vacuum field as it has been used in a closely related situation [9]. For the unobserved case the $N$ emitters are stimulated by the same vacuum, imparting phase correlations between them. On the observed system the pieces are influenced by different and statistically independent vacuum fields leading to mutually incoherent emissions. This heuristic picture refers to the idler instead of the signal field. However, it has been shown that a truly induced emission of different idler modes by mutually coherent fields leads to the emission of mutually coherent signal beams which otherwise are incoherent [10]. In fact, this induced coherence has also been observed in the case of no induced emission, or emission induced by the vacuum, in a situation closely related to the one studied here [11]. We can regard this effect as caused by the possibility which offers this configuration to control the signal beam by means of the idler one due to the strong quantum correlations established between them by this process.

Alternatively, the probability of emission on the unobserved system can be considered as the constructive interference between $N$ possible and intrinsically indistinguishable ways for the emission to occur. When we interrupt the idler path $N$ times, these ways become distinguishable by the possible detection of the idler photon. This possibility wipes out the interference, and the emission is modified. Modification of the spontaneous emission by changing the surrounding environment of the parametric down-conversion has also been observed as an interference effect in Ref. [12].

The dependence of the probability of emission of the signal photon on $N$ in (7) is the one characteristic of the Zeno effect. This parameter $N$ is the relative frequency of observation and also its accuracy. The complete inhibition of the emission only occurs in the limit $N \rightarrow \infty$ which can be considered as the limit of infinitely frequent or continuous observation. In our context it would correspond to an exact knowledge of the moment of emission. In the previous example, as in other situations, this kind of limit is only expressing a trend, but otherwise it could be void of meaning. This can be not only from practical reasons but also because for high $N$ the situation can change and other facts should be taken into account [4].

In what follows we study another example of Zeno effect in parametric down-conversion which is controlled by a more accessible parameter like it is an intensity. Instead of dividing the crystal into pieces, let us assume that in addition to the interaction described by the Hamiltonian (1) there is another interaction between the idler mode and another field which we will describe by the complex amplitude operator $b$. We are going to assume that this interaction is of the Kerr type, and we replace (1) by the interaction Hamiltonian

$$
\tilde{H}=\hbar g\left(a_{s}^{\dagger} a_{i}^{\dagger}+a_{s} a_{i}\right)+\hbar \kappa a_{i}^{\dagger} a_{i} b^{\dagger} b,
$$

$L$ being again the length of the crystal, and the associated interaction time is $\tau$. Now, the information concerning the moment of emission of the signal photon is contained in the phase of the mode $b$. Assuming that, in accordance with the short time approximation, only one pair of signalidler photons is produced, the phase of the field $b$ will be proportional to the length covered by the idler photon since it has been emitted or, equivalently, proportional to the time spent by the signal photon within the crystal. The instant of emission of the signal photon can then be inferred by a phase dependent measurement, such as homodyne or heterodyne detection, on the output field $b$. Here, only one measurement is enough instead of the $N$ measurements of the previous example.

Next, we examine whether this scheme disturbs the emission of the signal photon or not. Assuming the short time approximation, we can solve the input-output relation in powers of $g \tau$ retaining just the first power. We then have

$$
\tilde{a}_{s}^{\prime} \simeq a_{s}-g \tau \frac{e^{i \kappa \tau b^{\dagger} b}-1}{\kappa \tau b^{\dagger} b} a_{i}^{\dagger} .
$$

We will consider that the input signal and idler beams are in vacuum and the field in mode $b$ is an arbitrary state $|\varphi\rangle$. Since in this approximation the probability of having more than one signal photon is negligible, we have from (10)

$$
\tilde{P}_{s}(1) \simeq\left\langle\tilde{a}_{s}^{\prime \dagger} \tilde{a}_{s}^{\prime}\right\rangle \simeq(g \tau)^{2}\left\langle\varphi\left|\left[\frac{\sin \left(\kappa \tau b^{\dagger} b / 2\right)}{\kappa \tau b^{\dagger} b / 2}\right]^{2}\right| \varphi\right\rangle .
$$

We can see that the probability of emission of the signal photon is always less than or equal to the probability of the isolated case, and it depends on the choice of the input state $|\varphi\rangle$ of the field in mode $b$. On the other hand, 
the accuracy of the inference of the emission time of the signal photon also depends strongly on the choice for $|\varphi\rangle$. This is because the resolution of a phase shift estimation after a single measurement is, under the best conditions, proportional to the intensity of $|\varphi\rangle$ [7]; the more intense $|\varphi\rangle$ is, the better is the resolution that can be achieved.

In the limit when $|\varphi\rangle$ tends to be the vacuum the probability tends to be that of the isolated dynamics of the parametric down-conversion (4). However, in this limit the output field in mode $b$ tends to provide no information about the time of emission. The emission is not disturbed, but it is not possible to infer from the output field when the emission has taken place and no Zeno effect occurs. In order to have a precise measurement of the phase shift a field $|\varphi\rangle$ with strong intensity is needed. However, in the limit of an intense field $b$, the probability (11) tends to zero and the emission is here again inhibited.

We can give a rough estimation of this relation between the probability of emission and the accuracy of the inference. The uncertainty $\Delta \phi$ on the phase shift estimation can be, under the best conditions, of the order $\Delta \phi \sim 1 / n$, where $n$ accounts for the photon number of the field $b$. Since $\Delta \phi=\kappa \Delta \tau$, where $\Delta \tau$ is the uncertainty of the inferred emission time, the sinc function in (11) depends, very roughly, on $\kappa \tau n \sim \tau / \Delta \tau$. A true inference of the moment of emission can occur only if $\tau / \Delta \tau \gg 1$, but in such a case the probability of emission tends to be practically zero.

In comparison with the previous scheme the intensity of the field $|\varphi\rangle$ is playing here the role of $N$ there. But this analogy refers only to its role in the resolution of the observation and not to the meaning of frequency in the measurement, since here only one measurement is sufficient to infer the moment of emission. The limit of continuous observation, in the sense of arbitrary high resolution $\tau / \Delta \tau$, is more attainable here than in other situations, since it is approached by increasing the intensity and not by increasing the frequency of repeated measurements.

Here again it does not matter whether the output field in mode $b$ is finally measured or not, the observed system is disturbed anyway. The inhibition of the emission is due to the alteration of the isolate dynamics produced by the changes that must be introduced on the system in order to make possible the observation of the intermediate stages of the evolution. Here the Kerr interaction in (9) accounts for this. Since $b^{\dagger} b$ is a constant of the motion, the last term in (9) can be regarded as an effective mismatch between the signal and the idler fields, that depends on the intensity of the field $b$. This mismatch is more explicitly illustrated if we express the idler amplitude $a_{i}$ as $a_{i}=$ $A_{i} \exp \left(-i \kappa t b^{\dagger} b\right)$, having $\left[A_{i}, b^{\dagger} b\right]=0$ and $\left[A_{i}, A_{i}^{\dagger}\right]=$ 1. The equations of motion for $a_{s}$ and $A_{i}$ are then the same as those that would be obtained from the time dependent effective interaction Hamiltonian arising just from the first term in (9) after the said replacement. It is known that the down-conversion is correspondingly impeded by increasing the phase mismatch as it is reflected in the usual form in (11), here in terms of a fluctuating mismatch. It is then also possible to interpret this inhibition in terms of the impeded constructive interference of probability amplitudes. Photon number measurements based on Kerrlike interactions do not disturb the photon number of the measured system, in this case in the idler mode. However, they unavoidably affect its phase, and the parametric down-conversion is very sensible to the phase relation between the signal and idler beams.

This action of the arrangement set for the observation on the observed system accounts, as in the previous example, for the inhibition of the emission of the signal photon for this Zeno effect.

A.L. acknowledges the support of a grant from the Dirección General de Investigación Científica y Técnica del Ministerio Español de Educación y Ciencia and the kind hospitality from the Optics Department of the Palacký University. J. P. acknowledges partial support of the Grant No. 202/96/0421 of Czech Grant Agency.

*On leave of absence from Departamento de Optica, Facultad de Ciencias Físicas, Universidad Complutense, Madrid, Spain.

${ }^{\dagger}$ Also at Joint Laboratory of Optics, Palacký University, and Physical Institute of Academy of Sciences, Olomouc, Czech Republic.

[1] B. Misra and E. C. G. Sudarshan, J. Math. Phys. 18, 756 (1977).

[2] R. J. Cook, Phys. Scr. T21, 49 (1988); W. M. Itano, D. J. Heinzen, J. J. Bollinger, and D. Wineland, Phys. Rev. A 41, 2295 (1990).

[3] T. P. Altenmüller and A. Schenzle, Phys. Rev. A 49, 2016 (1994).

[4] H. Nakazato, M. Namiki, S. Pascazio, and H. Rauch, Phys. Lett. A 199, 27 (1995); A. Venugopalan and R. Ghosh, Phys. Lett. A 204, 11 (1995).

[5] P. Kwiat, H. Weinfurter, T. Herzog, A. Zeilinger, and M. A. Kasevich, Phys. Rev. Lett. 74, 4763 (1995).

[6] S. Pascazio and M. Namiki, Phys. Rev. A 50, 4582 (1994).

[7] J. Peřina, Z. Hradil, and B. Jurčo, Quantum Optics and Fundamentals of Physics (Kluwer Academic, Dordrecht, 1994).

[8] J. Peřina, Quantum Statisics of Linear and Nonlinear Optical Phenomena (Kluwer Academic, Dordrecht, 1991), 2nd ed.

[9] M. O. Scully and U. W. Rathe, Opt. Commun. 110, 373 (1994).

[10] L. J. Wang, X. Y. Zou, and L. Mandel, J. Opt. Soc. Am. B 8, 978 (1991).

[11] X. Y. Zou, L. J. Wang, and L. Mandel, Phys. Rev. Lett. 67, 318 (1991).

[12] T. J. Herzog, J. G. Rarity, H. Weinfurter, and A. Zeilinger, Phys. Rev. Lett. 72, 629 (1994); T. J. Herzog, P. G. Kwiat, H. Weinfurter, and A. Zeilinger, Phys. Rev. Lett. 75, 3034 (1995). 